\title{
Article \\ Facilitating Machine Learning Model Comparison and Explanation through a Radial Visualisation ${ }^{\dagger}$
}

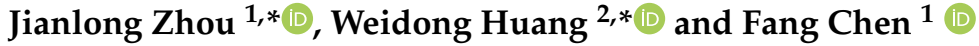 \\ 1 Data Science Institute, University of Technology Sydney, Ultimo, NSW 2007, Australia; fang.chen@uts.edu.au \\ 2 TD School, University of Technology Sydney, Ultimo, NSW 2007, Australia \\ * Correspondence: jianlong.zhou@uts.edu.au (J.Z.); weidong.huang@uts.edu.au (W.H.) \\ $+\quad$ This paper is an extended version of our paper published in 2020 IEEE Pacific Visualization Symposium \\ (PacificVis), Tianjin, China, 3-5 June 2020; pp. 226-230.
}

Citation: Zhou, J.; Huang, W.; Chen, F. Facilitating Machine Learning Model Comparison and Explanation through a Radial Visualisation. Energies 2021, 14, 7049. https:// doi.org/10.3390/en14217049

Academic Editors: Valentina Colla and Jaroslaw Krzywanski

Received: 23 September 2021

Accepted: 21 October 2021

Published: 28 October 2021

Publisher's Note: MDPI stays neutral with regard to jurisdictional claims in published maps and institutional affiliations.

Copyright: (c) 2021 by the authors. Licensee MDPI, Basel, Switzerland. This article is an open access article distributed under the terms and conditions of the Creative Commons Attribution (CC BY) license (https:// creativecommons.org/licenses/by/ $4.0 /)$.

\begin{abstract}
Building an effective Machine Learning (ML) model for a data set is a difficult task involving various steps. One of the most important steps is to compare a substantial amount of generated ML models to find the optimal one for deployment. It is challenging to compare such models with a dynamic number of features. Comparison is more than only finding differences of ML model performance, as users are also interested in the relations between features and model performance such as feature importance for ML explanations. This paper proposes RadialNet Chart, a novel visualisation approach, to compare ML models trained with a different number of features of a given data set while revealing implicit dependent relations. In RadialNet Chart, ML models and features are represented by lines and arcs, respectively. These lines are generated effectively using a recursive function. The dependence of ML models with a dynamic number of features is encoded into the structure of visualisation, where ML models and their dependent features are directly revealed from related line connections. ML model performance information is encoded with colour and line width in RadialNet Chart. Taken together with the structure of visualisation, feature importance can be directly discerned in RadialNet Chart for ML explanations. Compared with other commonly used visualisation approaches, RadialNet Chart can help to simplify the ML model comparison process with different benefits such as the following: more efficient in terms of helping users to focus their attention to find visual elements of interest and easier to compare ML performance to find optimal ML model and discern important features visually and directly instead of through complex algorithmic calculations for ML explanations.
\end{abstract}

Keywords: machine learning; performance; bar chart; line chart; radar chart; RadialNet chart; visualisation

\section{Introduction}

We have witnessed a rapid boom of data in recent years from various fields such as infrastructure, transport, energy, health, education, telecommunications, and finance. Together with the dramatic advances in Machine Learning (ML), obtaining insights from these "Big Data" and data analytics-driven solutions are increasingly in demand for different purposes. While "Big Data" is used by sophisticated ML algorithms to train ML models which are then evaluated by various metrics such as accuracy, the generated substantial amounts of ML models must be compared by the engineering designers and analysts to find the optimal one for deployment. Figure 1 shows a typical pipeline that processes data to find an optimal ML model. Taking a data set with multiple features for ML training as an example, multiple features can be grouped differently as the input for an ML algorithm to train different ML models. For example, if a data set has three features of F1, F2, and F3, these features may have seven different groups: (F1), (F2), (F3), (F1, F2), (F1, F3), (F2, F3), and (F1, F2, F3). Each feature group can be used as the input for an ML algorithm to train an ML model, thereby obtaining seven different ML models. It is a common thread 
to find the best/worst model by comparing such models; however, it is often challenging when having a large number of features. Furthermore, comparison is more than only finding differences of ML model performance, as users are also interested in the relations between features and model performance from comparison in order to obtain explanation of models, for example, to find which features result in high performance of ML models, and those features are referred as high important features or vice versa. This is because the identifications of the most or least important features are the key steps for feature engineering in effective and explainable machine learning.

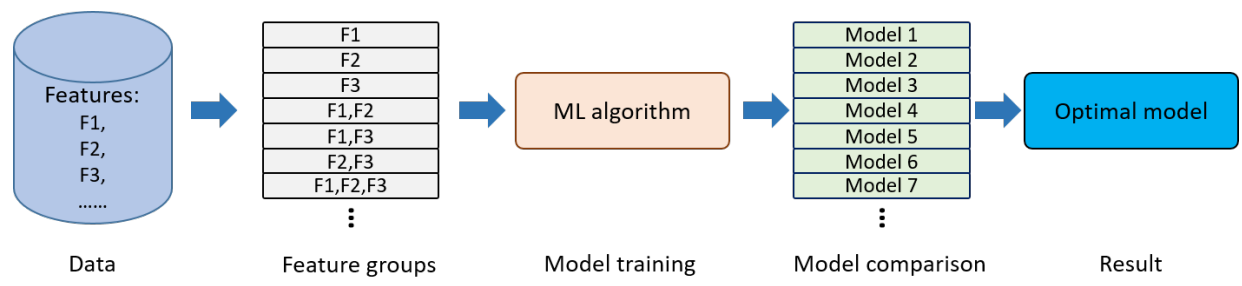

Figure 1. The pipeline of obtaining an optimal ML model for a data set with multiple features. Reprint with permission from Jianlong Zhou, Weidong Huang and Fang Chen (2020). Copyright 2020 IEEE Pacific Visualization Symposium (PacificVis).

On the other hand, it is widely recognised that visualisations amplify human's cognition during data analysis [1] and proper visualisation of ML outcomes is essential for a human analyst to be able to interpret them [2-4]. Viegas and Wattenberg [5] claimed that "data visualisation of the performance of algorithms for the purpose of identifying anomalies and generating trust is going to be the major growth area in data visualisation in the coming years". More importantly, comparison with visualisation is imperative for identifying the optimal model from substantial amounts of ML models. Bar charts, radar charts, line charts, and others [6] are commonly used visualisation methods in machine learning for comparing different variables. However, the comparison of ML models with a large number of features is still considered challenging with the aid of these commonly used visualisations: The items for comparison and the relationships between them can be highly complicated. These commonly used visualisation approaches not only cause information clutters for large number of visual elements (e.g., bars, dots, and lines) but also miss relation information between features and models, which are significant in ML explanations. It is also very difficult for users to differentiate differences between various models' performances with these commonly used visualisation approaches. Despite the specific focus on visualising comparison in recent studies [7-10], little work has been conducted on the visual comparison of ML models while identifying relations between features and ML models (e.g., the most and least important features).

We explore an approach based on the structure of visualisation in addressing challenges of comparison ML models with a dynamic number of features: while height information of bars and lines in commonly used visualisation approaches only encode one-dimensional information in a 2-dimensional (2D) space, it is possible to encode ML model information in other dimensions of the space. If both visual elements and structure of visualisation can be used to encode information of ML models, insights about ML models could be automatically generated, and users would not have to inspect every model to find the optimal one or conduct complex calculations to estimate feature importance.

In this paper, we propose RadialNet Chart (also referred to RadialNet in this paper), a novel visualisation approach for comparing ML models with different number of features while revealing implicit dependent relations. In RadialNet, ML models and features are represented by lines and arcs, respectively (an arc also represents the model based on a single feature of arc). The challenge of revealing dependence of ML models with a dynamic number of features is addressed by encoding such information into the structure of visualisation, where ML models and their dependent features are directly revealed from related line connections. These lines are defined by using a recursive function to 
generate them effectively. ML model performance information is encoded with colour and line width in RadialNet. It simplifies the comparison of different ML models based on these visual encoding. Moreover, together with the structure of visualisation, feature importance can be directly discerned in RadialNet for ML explanation. RadialNet uses a concept of feature path for ML model lines to avoid a large number of line entanglement. Moreover, when visual elements for ML models become crowded, RadialNet allows interactively changing the spanning space that RadialNet covers in order to dynamically control the visual complexities. To understand the effectiveness of RadialNet, we conducted a comparison experiment with three commonly used visualisation approaches of line chart, bar chart, and radar chart. The comparison experiment was evaluated with eleven researchers and developers experienced in machine learning related areas. The findings show that RadialNet has advantages in identifying features related to specific models as well as directly revealing the importance of features (for ML explanations). Furthermore, RadialNet is more efficient in helping users focus their attention to find visual elements of interest. It is more compact to show more information in a limited space compared with other visualisation methodologies.

This paper is the extended version of the conference paper of [10]. This extended version includes a detailed literature review with more related works, more detailed information about the methodology, and detailed implementation of RadiaNet. It also includes an extensive evaluation of the proposed visualisation approach with user studies for additional insights.

\section{Background and Related Work}

In machine learning, given a fixed number of features, it is possible to use different features and their groups to train machine learning algorithms resulting in various machine learning models. Users need to compare these models to find the optimal one for their tasks. Obtaining optimal results out of machine learning models requires truly understanding all models. However, each data set with a large number of features can have hundreds or even thousands of ML models, rendering it nearly impossible to understand all models based on different feature groups in an intuitive fashion. Visualisation can be used to help unlock nuances and insights in ML models.

This section investigates various visualisations from the perspectives of multi-attribute data visualisation, visualisation in explanation of machine learning, and comparison visualisation in order to demonstrate the state-of-art approaches and challenges for the comparison of machine learning models with visualisation.

\subsection{Visualisation of Multi-Attribute Data}

The comparison of the visualisation of machine learning models is related to multiattribute (or multiple features) data visualisation. The visualisation of multi-attribute data has been frequently investigated for years [11]. For example, multidimensional projections are one of the effective methods for visualizing high-dimensional data sets in order to find structures in the data such as groups of similar points and outliers. One of classical approaches for visualizing multi-attribute data points is the use of parallel coordinates [12]. The advantage of this technique is that it provides an overview of data trend. One of obvious disadvantages of parallel coordinates is that it lacks a tabular view for presenting value details of each coordinate. SimulSort [13] organized different attributes of data in a tabular and sorts all of the attribute columns simultaneously. However, users still need laborious interactions in SimulSort in order to highlight different points for comparison. Zhou et al. [14] proposed a visualisation approach for presenting multi-attribute data by combining advantages of both parallel coordinates and SimulSort, which organizes various attributes in a tabular-like form implicitly. Colours are used to encode data belonging to different groups, instead of highlighting attributes of one point at a time as in SimulSort. Such colour encoding approach provides an overview of points and their associated attribute details to improve information browsing efficiency. Motivated by such colour 
encoding, this paper uses colours to encode ML model performance in order to provide an overview of performance for comparison. However, such visualisation cannot reveal the complex relations between machine learning models and their dependent features with dynamic numbers.

Moreover, the contradiction between limited space and the large amount of information to be presented is another challenge for multi-attribute data visualisation. Coordinated and multiple views (CMV) [15] is widely used to extend the limited space of a single view for large data set visualisation. Langner et al. [16] presented a framework that uses a set of mobile devices to distribute and coordinate multiple visualisation views for the exploration of multivariate data. Koytek et al. [17] proposed MyBrush for extending brushing and linking technique by incorporating personal agency in the interactive exploration of data relations in CMV. Sarikaya et al. [18] introduced a framework to help determine the design appropriateness of scatterplot for task support to modify/expand the traditional scatterplots to scale as the complexity and amount of data increases. Most of these investigations focus on the extension of spaces for the complex information presentation; however, they ignore making full use of a given limited space. Our approach in this paper aims to encode complex information with less visual elements (e.g., model lines) in order to avoid entangled visual elements in the limited space and to improve information presentation efficiency.

\subsection{Visualisation in Explanation of Machine Learning}

Yuan et al. [19] reviewed techniques of visual analytics for machine learning by categorising them into techniques before model building, techniques during modeling building, and techniques after model building. Chatzimparmpas et al. [20] investigated approaches of enhancing trust in ML models with the use of interactive visualization. Visualisation is also used in ML explanations. Corresponding to the term of Exploratory Data Analysis (EDA) in terms of the desired outcome of the analytic process, Cashman et al. [21] presented a concept of Exploratory Model Analysis (EMA) with a user-based visual analytics workflow, which is defined as the process of discovering and selecting relevant models that can be used to make predictions on a data source. However, it does not consider the comparison of models with a different number of features.

In the early years, visualisation is primarily used to explain the ML process of simple ML algorithms in order to understand the ML process. For example, different visualisation methods are used to examine specific values and show probabilities of picked objects visually for Naïve Bayes [2], decision trees [22], and Support Vector Machines (SVMs) [23]. Advanced visualisation techniques are then proposed to present more complex ML processes. Erra et al. [24] introduced visual clustering that utilised a collective behavioral model, where visualisation helps users to understand and guide the clustering process. Paiva et al. [25] presented an approach that employs similarity tree visualisation to distinguish groups of interest within the data set. Visualisation is also used as an interaction interface for users in machine learning. For example, Guo et al. [26] introduced a visual interface named Nugget Browser allowing users to interactively submit subgroup mining queries for discovering interesting patterns dynamically. EnsembleMatrix allows users to visually ensemble multiple classifiers together and provides a summary visualisation of results of these multiple classifiers [3]. Zhou et al. [27] revealed states of key internal variables of ML models with interactive visualisation in order to allow users to perceive what is processes are occurring inside a model.

More recent work tries to use visualisation as an interactive tool to facilitate ML diagnosis. ModelTracker [28] provides an intuitive visualisation interface for ML performance analysis and debugging. Chen et al. [29] proposed an interactive visualisation tool by combining ten state-of-the-art visualisation methods in ML (shaded confusion matrix, ManiMatrix, learning curve, learning curve of multiple models, McNemar Test matrix, EnsembleMatrix, Customized SmartStripes, Customized ModelTracker, confusion matrix with sub-categories, and force-directed graph) in order to help users interactively carry 
out a multi-step diagnosis for ML models. Wongsuphasawat et al. [30] presented the TensorFlow Graph Visualizer to visualise data flow graphs of deep learning models in TensorFlow to help users understand, debug, and share the structure of their deep learning models.

Visualisations comprise the major body of ML process explanations. However, these approaches cannot be directly used for the comparison of machine learning models trained with a different number of features nor facilitate the revelation of feature importance directly from visualisations of models for ML explanations.

\subsection{Comparison Visualisation}

Supporting comparisons is a common challenge in visualisation. Gleicher [7] categorized four considerations that abstract comparison when using visualisation. These four considerations included identifying the following: the comparative elements, the comparative challenges, a comparative strategy, and a comparative design, which provide a guideline for developing comparison solutions in visualisation. Law et al. [8] presented Duet, a visual analysis system for conducting pairwise comparisons. Duet employs minimal specification in comparison by only recommending similar and different attributes between them when one object group to be compared is specified. Qi et al. [31] presented a visual technique called STBins for visual tracking of individual data sequences and also for the comparison of multiple sequences. The comparison of sequences is performed by showing the similarity of sequences within temporal windows. The analysis of subtle deviations between different versions of historical prints is important but also a challenge in art history research. Plüger et al. [32] developed an approach called VeCHart that detects similar stroke-patterns in prints and matches them in order to allow visual alignment and automated deviation highlighting for comparison purposes. Cutura et al. [33] proposed a visual analysis approach called Compadre for comparing the distances of high-dimensional data and their low-dimensional projections. The key to visual analysis is a matrix visualization for representing the discrepancy between distance matrices, which are linked with 2D scatter plot projections of the data. Heimerl et al. [34] introduced an interactive visualisation approach of embComp for comparing two embeddings that capture the similarity between objects, such as word and document embeddings. The proposed approach features overview visualizations that are based on metrics for measuring differences in the local structure around objects and detailed views allowing comparisons of the local structure around the selected objects and relating this local information to global views. However, little work is performed on the comparison of machine learning with a different number of features.

The bar chart is one of commonly used visualisation methods for comparison in machine learning [6]. It works with two variables-one is the length of the bar on one axis and the second is the position of this bar on another axis. The variable is compared by denoting it with the length of the bars when various bars are plotted together. Radar Chart is another commonly used approach for comparing multiple quantitative variables. It is useful for observing which variables have similar values or if there are any outliers amongst the values of each variable. It can also help to find which variables are high or low. Moreover, other methods such as line chart and ring chart are also used in comparison. Ondov et al. [9] made evaluations of comparison visualizations of five layouts: stacked small multiples, adjacent small multiples, overlaid charts, adjacent small multiples that are mirror symmetric, and animated transitions. The data to be compared are encoded with the length of bars in bar charts, slop of lines in line charts, and angle of arcs in donut charts.

These previous works provide significant guidelines and advances in comparison visualisation. This paper proposes a new visualisation method for machine learning model comparison with full considerations of four aspects as categorized in [7]. The new visualisation approach is evaluated by comparing it with other three commonly used visualisation methods (bar chart, line chart, and radar chart) in machine learning model comparisons. 


\section{RadialNet Chart}

This section presents a novel visualisation approach called RadialNet Chart in order to compare machine learning models trained with different feature groups of a data set.

\subsection{Design Goals}

After conducting a thorough survey with experienced researchers and developers in machine learning on their problems in comparing machine learning models, we phrase the following design goals for RadialNet:

- Comparison: To maximise differences among visual elements of models in order to help users find the optimal target easily. The comparison is the core objective in the ML model visualisation. This is a challenge when substantial amounts of ML models must be compared.

- Importance: To easily identify the importance of features directly from visualisation. The importance of features plays significant roles in the feature selection in the ML pipeline and ML explanations [35]. It is a challenge to identify the importance of features directly from visualisation without complex feature importance calculations.

- Feature identification: To easily identify relationships between models (and model performance) and their dependent features. This helps users easily link ML models and their dependent features for understanding both features and models, which is usually challenging with commonly used visualisation approaches.

- Compactness: To represent complex visualisation in a compact form and reduce visual clutters because of substantial amounts of information in a limited space.

\subsection{Definition of RadialNet Chart}

This subsection defines the RadialNet. Figure 2 shows an example of RadialNet. Based on this example, we firstly provide the following definitions that are used to set up RadialNet:

Feature arc Each feature is represented by a concentric arc in RadialNet. The arc is also called feature arc. The name of each feature is displayed at one end of the arc, as shown in Figure 2 (e.g., F1, F2, F3, and F4). Each arc also represents the ML model based on that single feature.

Model line RadialNet uses a line segment to represent an ML model based on multiple features. The line is also called model line. For example, in Figure 2, the line $A B, B C$, and $C D$ represent different ML models, respectively. The features used for the model are defined based on the feature path of the line (refer to the definition of feature path below).

Feature point A feature point refers to an intersection point of a model line with an arc. It is represented by a dot point on a feature arc as shown in Figure 2 (e.g., feature points $\mathrm{A}, \mathrm{B}$, and $\mathrm{C}$ ).

Feature path A feature path defines features used for a model line. A feature path starts from the feature point of a model line on its outermost arc and ends at the feature point on the innermost arc it can reach through the connected feature point in the RadialNet. For example, in Figure 2, for the model line $A B$, its feature path starts from feature point A on the arc F4, passes through B and C, and ends at D on the innermost arc F1. This path can be represented by a list of features corresponding to the arcs of each feature point, i.e., the feature path of $A B$ is (F4, F3, F2, F1). Similarly, the feature path of $B C$ is (F3, F2, F1), the feature path of $C D$ is $(F 2, F 1)$, the feature path of $E C$ is $(F 4, F 2, F 1)$, the feature path of $M P$ is $(F 4, F 3, F 2)$, and the feature path of $P Q$ is $(\mathrm{F} 3, \mathrm{~F} 2)$.

Furthermore, model performance is encoded by using two methods: the width of the line/arc and the colour of the line/arc. The wider the line/arc is, the higher the model performance. A colour scale is accompanied with the RadialNet to encode model 
performance and allows users to easily perceive the difference of performance of the different models, as shown in Figure 2.

Based on these definitions, the visualisation of lines and arcs spirals from the centre to the outside area; therefore, it is called RadialNet Chart. The RadialNet has different advantages. For example, given a data set in machine learning, if most of ML models related to one specific feature show high model performance, that feature can be considered as a high important feature; in the vice versa case, if most of ML models related to one specific feature show low model performance, that feature can be considered as a less important feature. RadialNet can depict the importance of features directly by visualisation: if an arc and its connected lines are mostly wider than others and have colours representing high performance values in the colour scale, the feature represented by the arc is an important feature; in the vice versa case, it can also depict less important features. For example, in Figure 2, feature F1 is an important feature because the width and colour of the arc as well as its connected lines are mostly wider and red, while feature F4 is a less important feature. The RadialNet also helps users in directly identifying features used for a specific model because of the feature path mechanism in RadialNet.

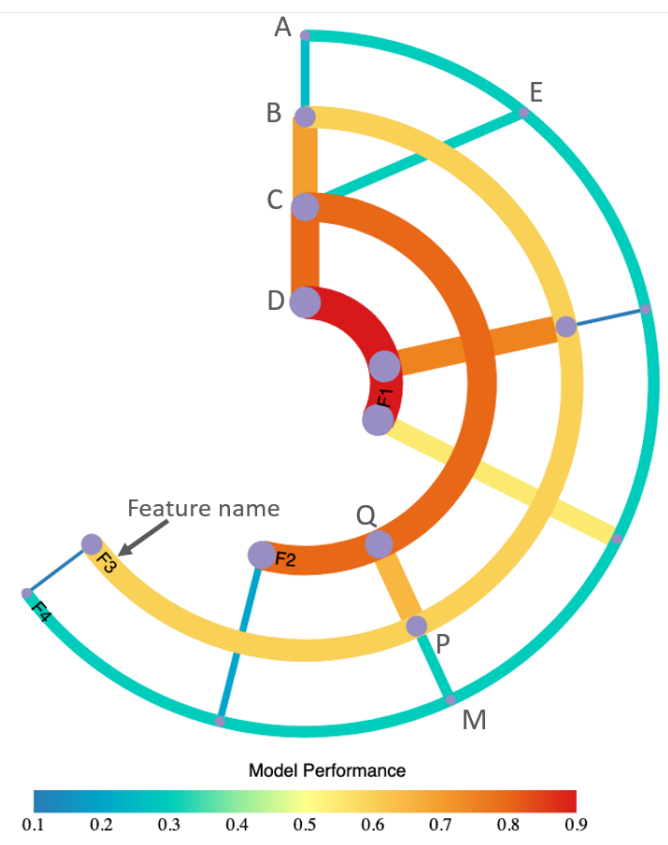

Figure 2. An example of RadialNet chart. Reprint with permission from Jianlong Zhou, Weidong Huang and Fang Chen (2020). Copyright 2020 IEEE Pacific Visualization Symposium (PacificVis).

Figure 3 shows the steps used to draw a RadialNet. The definition of different parameters is the key during RadialNet drawing. Firstly, key parameters are defined with user interactions or predefined approaches. Arc parameters and line parameters are then generated based on key parameters. The RadialNet is drawn finally based on generated parameters.

Key parameter
initialization $\Rightarrow \begin{gathered}\text { Arc parameter } \\ \text { generations }\end{gathered} \Rightarrow \begin{gathered}\text { Line parameter } \\ \text { generations }\end{gathered} \Rightarrow \begin{gathered}\text { Spiral chart } \\ \text { drawing }\end{gathered}$

Figure 3. The steps for drawing RadialNet. Reprint with permission from Jianlong Zhou, Weidong Huang and Fang Chen (2020). Copyright 2020 IEEE Pacific Visualization Symposium (PacificVis).

\subsection{Key Parameter Initialization}

The key parameters include the overall spanning angle of RadialNet, the overall number of models given the number of features, and the size of the drawing canvas, as 
well as others. The overall spanning angle defines the space that the RadialNet covers in degrees. It can be interactively modulated by users to control the compactness of the visualisation in a limited space. If the number of ML models to be visualized is low, a small value can be defined for the spanning angle; in the vice versa case, a large value can be defined for the spanning angle in order to help users to easily control and compare ML models in a limited space.

Given $N$ features of a data set, F1, F2, ..., FN, a machine learning algorithm uses these features to set up ML models. The ML models can be set up based on one or multiple features of the data set. Typically, the number of models based on various groups of $N$ features can be obtained from Equation (1):

$$
C_{N}=C_{N}^{1}+C_{N}^{2}+\ldots+C_{N}^{i}+\ldots+C_{N}^{N}=2^{N}-1
$$

where $C_{N}$ is the number of models based on groups of $N$ features, and $C_{N}^{i}$ is the group number of selecting $i$ features from $N$ features. It shows that the number of ML models is increased exponentially with the increase in the number of features.

Furthermore, because of the circular characteristics of RadialNet, polar coordinates are used to represent arcs and lines in RadialNet.

\subsection{Arc Parameter Generations}

Algorithm 1 shows the process for generating arc parameters. The arc is denoted by its start point and end point in polar coordinates. In this algorithm, arcSpanning defines the largest angle that arcs cover in the space and can be interactively changed by a sliding bar in the user interface. $N$ is the number of features. canvasWidth is the width of the drawing canvas. allFeatures is a list of all studied features which are sorted in the decreased order based on model performance of individual features. Each arc represents the model performance based on an individual feature from allFeatures list. The algorithm generates arc parameters aiming to make $N$ arcs evenly distributed in the drawing canvas space. This algorithm initializes the spanning angle of each arc with the arcSpanning value, and the spanning of each arc (arcAngle) is dynamically updated in the drawing algorithm (refer to Algorithm 3) in order to allow arcs in a spiral format. arcParasDict is a dictionary storing parameters of arcs, and the key of the dictionary is the individual features for the arc. The parameters include the arc's radius, spanning angle, and arc width. Data are read from a JSON file and stores different feature groups and their model performance values.

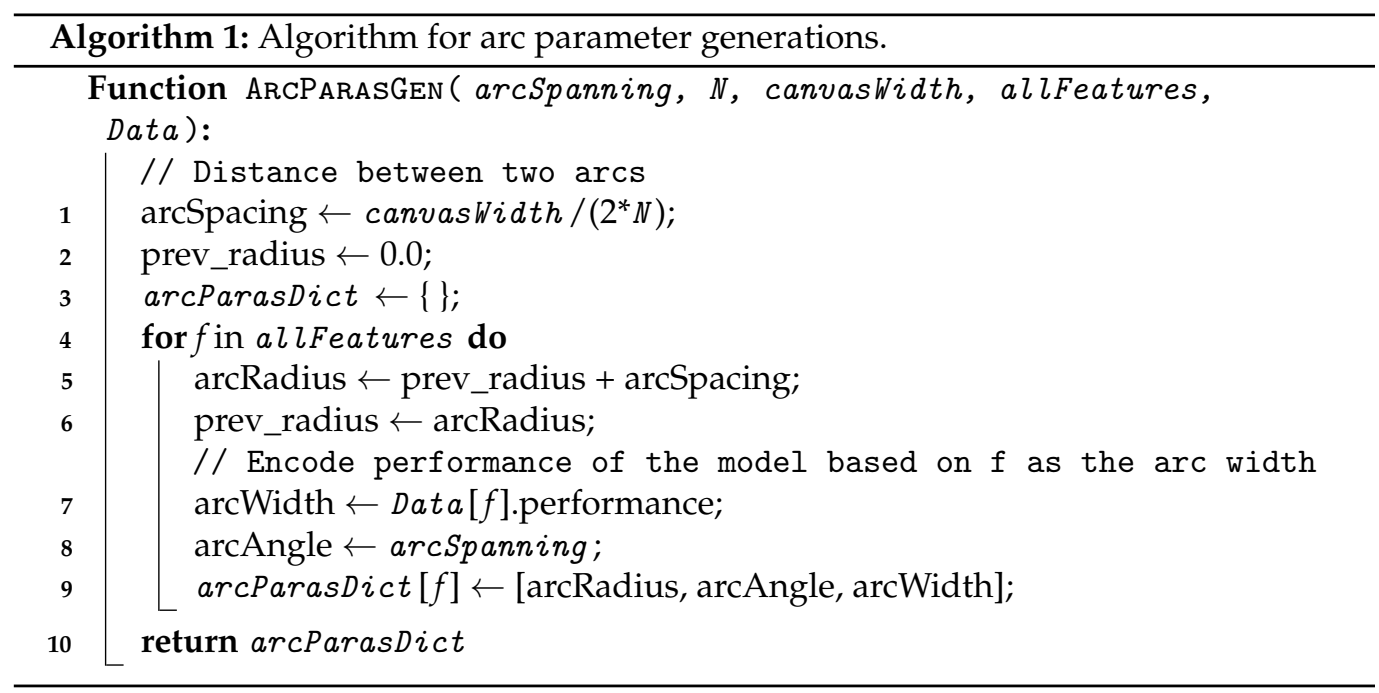




\subsection{Line Parameter Generations}

Algorithm 2 shows a recursive function used for generating model line parameters. The line is denoted by its start point and end point in polar coordinates. In this algorithm, lineParasDict is a dictionary and stores parameters of lines, and the key of the dictionary is the feature list (feature path) used for the line. The line parameters stored in the dictionary include the start and end points of the line in polar coordinates as well as line width of the line. lineFeatures is the feature list for the current line and is sorted in decreasing order based on the model performance of individual features. startangle is the angle of polar coordinates of the start point of the line. angleStep is the step size that angle increases each time.

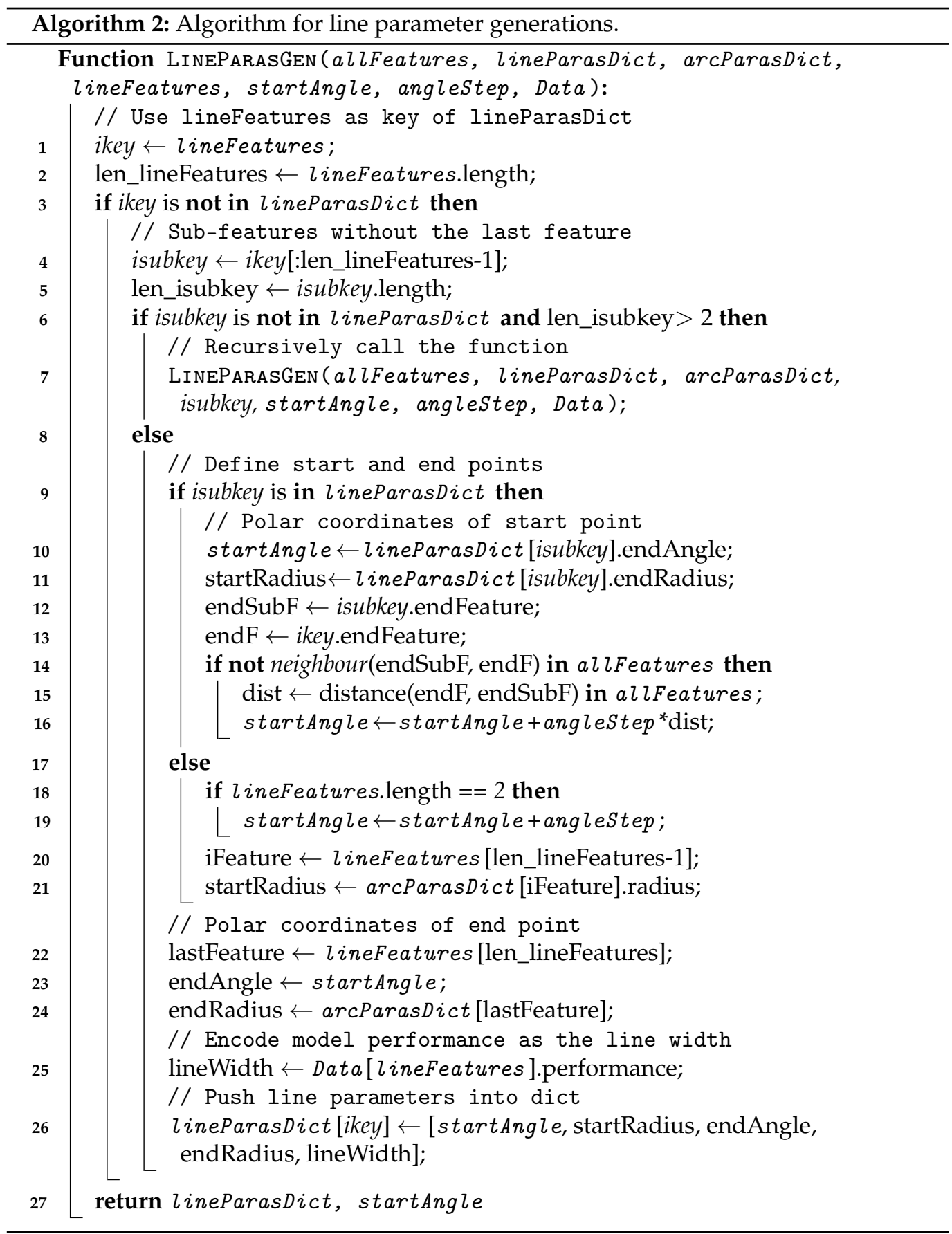

In this algorithm, if the key with the current lineFeatures does not exist in lineParasDict, a sub-key with the feature list by removing the last feature in lineFeatures is created. 
If this sub-key still does not exit in lineParasDict and the number of features in this subkey is more than two, the algorithm recursively calls this function with the current sub-key features. Otherwise, the algorithm defines the start point and end point of the line and pushes them into lineParasDict.

The line width is encoded with model performance based on lineFeatures. The colour of the line is also encoded with model performance by using a colour scale.

\subsection{RadialNet Chart Drawing}

Algorithm 3 shows the process of drawing a RadialNet. In Algorithm 3, after obtaining key parameters such as the number of points on the outermost arc and arc spanning angle, Algorithm 1 is firstly called to generate arc parameters. Then, Algorithm 2 is called for each feature to generate line parameters related to that feature. These parameters are then used to draw arcs and lines by calling functions of DrawArcs() and DrawLines(), respectively. DrawArcs() and DrawLines() calls Javascript functions in order to draw arcs and lines.

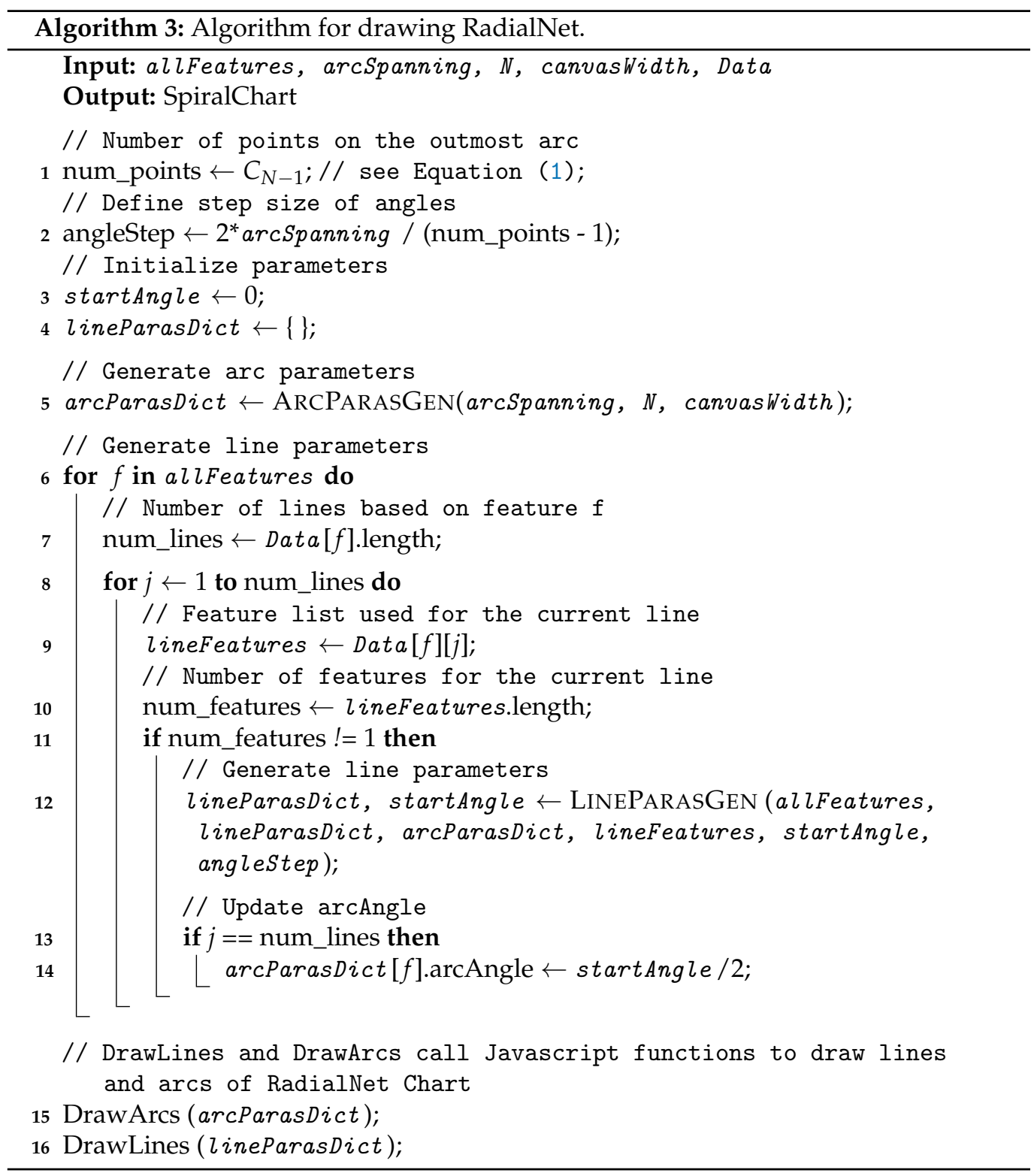

\section{Implementation}

The proposed approach is implemented in Javascript based on the D3.js library [36]. The data inputs relative to RadialNet are saved in a JSON file. RadialNet is also imple- 
mented as a Javascript library, and it is can easily be reused in different visualisation applications. This library will be released as an open source library.

\section{Case Studies}

In this section, RadialNet is used to visualise machine learning models based on different data sets and ML algorithms. Two data sets from UCI machine learning data repository [37] and PPMI [38], respectively, were analyzed, and three machine learning algorithms of K-Nearest Neighbours (KNN), Naïve Bayes (NB) and Random Forest (RF), were deployed in the experiment. Figure 4 shows the visualisation of different ML models for a data set with six features. From this visualisation, we can easily locate the model with the highest performance (the widest red line $A B$ as shown in Figure 4) as well as features

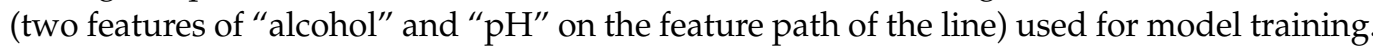
It also helps users to easily identify the importance of features, and the most important feature "alcohol" is represented by the outermost arc (the arc and its connected lines are mostly redder and wider than others) while the least important feature "free suffur" is represented by the innermost arc (the arc and its connected lines are mostly bluer and narrower than others). Figure 5 shows the visualisation of different ML models for a data set with seven features. Compared with Figure 4, the model number increased dramatically when the feature number is increased by only one. This visualisation also helps users to easily locate the model with the lowest performance (the narrowest blue line $A B$ as shown in Figure 5). We can also easily directly identify the most important feature (the third inner arc represented by the widest red arc) and the least important feature (the innermost narrowest yellow arc), as shown in Figure 5.

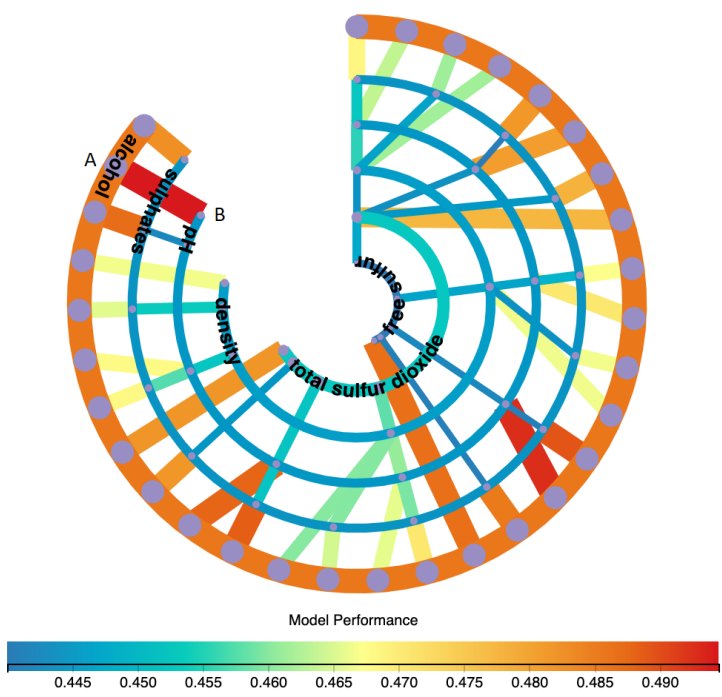

Figure 4. RadialNet of ML models based on a data set with 6 features. Reprint with permission from Jianlong Zhou, Weidong Huang and Fang Chen (2020). Copyright 2020 IEEE Pacific Visualization Symposium (PacificVis).

In addition to the comparison of feature importance of a data in RadialNet, it can also be used to compare performance of different ML algorithms for a given data set. Figure 6 shows the comparison of three ML algorithms for the same data set with RadialNet visualisation. From this figure, we can easily evaluate that the ML algorithm represented by the left diagram shows the worst performance compared to algorithms represented by the other two diagrams because its colour is more blue, which is located on the left side of the colour scale, while the algorithm represented by the middle diagram shows the best performance because its colour is more red, which is located on the right side of the colour scale. Furthermore, the visualisation shows that the feature represented by the outermost arc (i.e., the feature of "alcohol") is the most important feature because this arc is the widest and its colour is located on the right side of the colour scale in all three visualizations. 


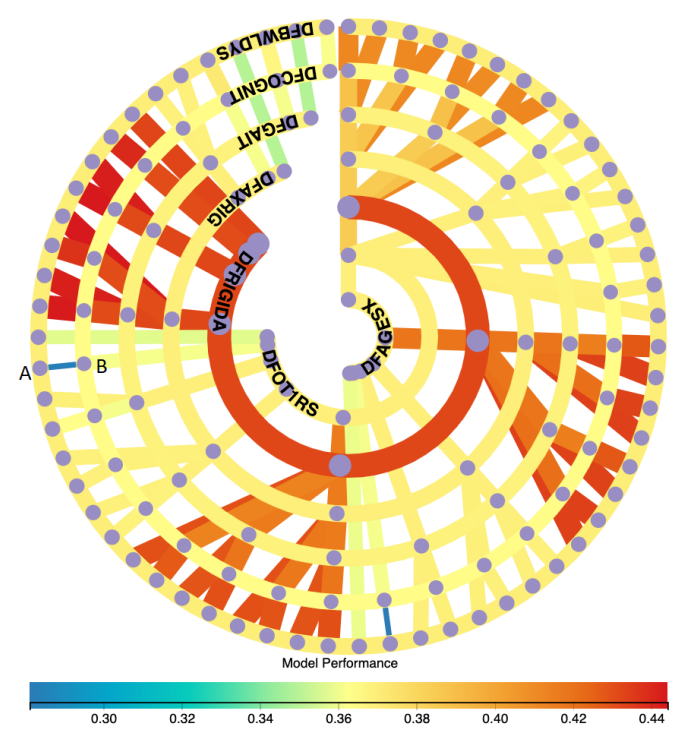

Figure 5. RadialNet of ML models based on a data set with 7 features. Reprint with permission from Jianlong Zhou, Weidong Huang and Fang Chen (2020). Copyright 2020 IEEE Pacific Visualization Symposium (PacificVis).
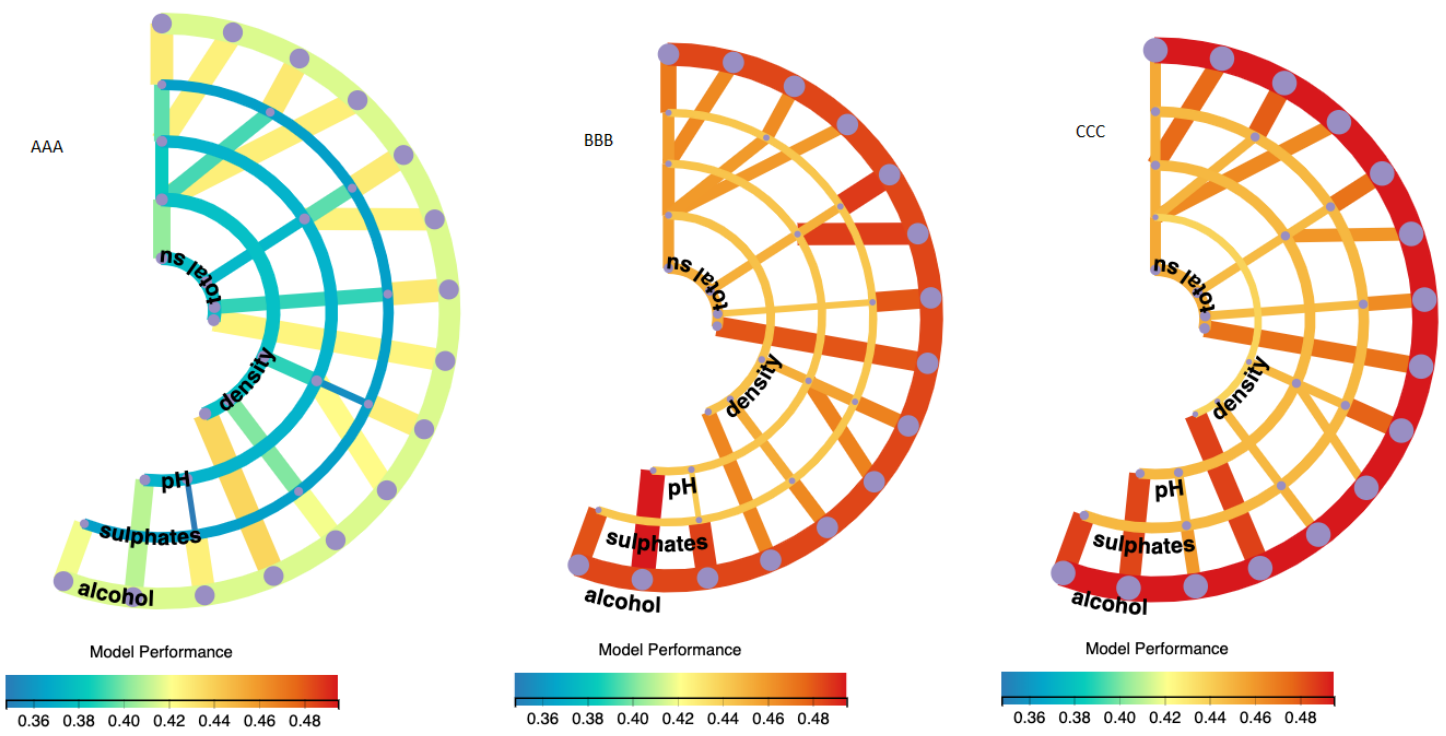

Figure 6. Comparison of three ML algorithms for the same data set with RadialNet. Reprint with permission from Jianlong Zhou, Weidong Huang and Fang Chen (2020). Copyright 2020 IEEE Pacific Visualization Symposium (PacificVis).

\section{Evaluation}

In order to understand the effectiveness of RadialNet in the ML model comparison, we compare it with three commonly used visualisation approaches of bar chart, line chart, and radar chart. Eleven participants were recruited (nine males and two females; ages from 20 to 40) in order to conduct a comparison user study. All participants are researchers and developers experienced in machine learning related areas.

The following metrics were proposed to evaluate different visualisations:

- Comparison: How easily can the visualisation help users to compare performance of different models;

- Feature importance: How easily can the visualisation help users in identifying the importance of features;

- Feature identification: How easily can the visualisation help users to link each model and its dependent features; 
- Complexity: How complex is the visualisation in terms of presenting data.

Moreover, user cognitive responses relative to visualisation such as mental effort and time spent on the selection task are also evaluated in order to compare the effectiveness of visualizations:

- Mental effort: How much mental effort users used for tasks with the visualisation;

- Time spent: How much time users spent in task decisions with the visualisation.

In order to understand the usability of the RadialNet Chart, we also administrated a questionnaire that asks participants questions about their experience and feedback in using the charts. Furthermore, an eye tracking study was conducted with a separate participant to understand the participant's eye movement behaviour with different visualisations [39].

\subsection{Data and Visualisation}

Two data sets from UCI machine learning data repository [37] and PPMI [38], respectively, were analysed in this study. The two data sets have six features and seven features, respectively, which generate $63 \mathrm{ML}$ models and $127 \mathrm{ML}$ models, respectively, for comparison. ML models are visualised by using bar chart, line chart, radar chart, and RadialNet, respectively, as shown in Figures 4 and 7 (the data set with six features visualised in Figures 4 and 7). In bar chart, line chart, radar chart and RadialNet, the related features for a model and its performance pop up when the mouse hovers over the relevant visual elements (e.g., bars, dots, lines, or arcs), which allows users to inspect more details of each model.

Moreover, for a given data set, three ML algorithms were used generating various ML models, respectively. The ML models by these three ML algorithms were visualised together in a single bar chart, line chart, and radar chart, respectively, as shown in Figure 8, which were also visualised using RadialNet as shown in Figure 6. These visualisations were used to compare the effectiveness of different ML algorithms. AAA, BBB, and CCC in visualisations (e.g., Figures 7 and 8) represent three ML algorithms used to compare KNN, NB, and RF. The exact ML algorithms used for ML models were not shown to participants during the study in order to avoid any bias.

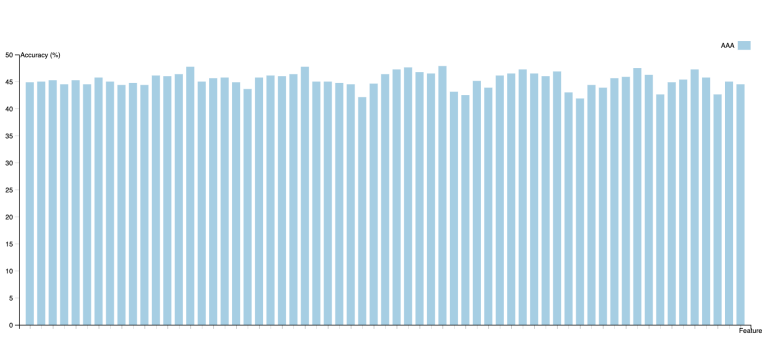

(a) Bar chart.

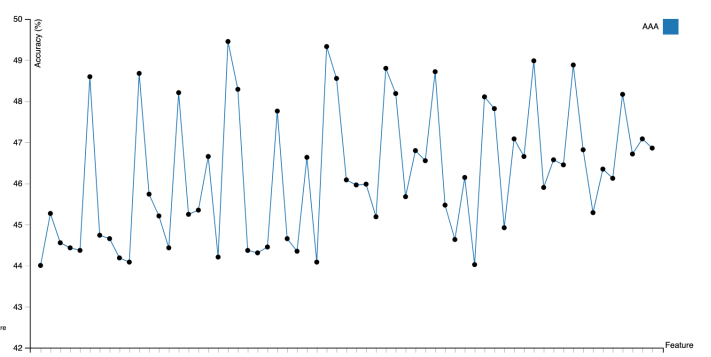

(b) Line chart.

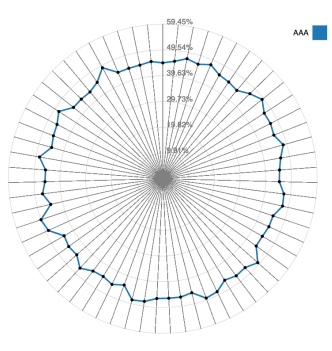

(c) Radar chart.

Figure 7. ML models based on the data set of 6 features are visualized using bar chart, line chart, and radar chart, respectively.

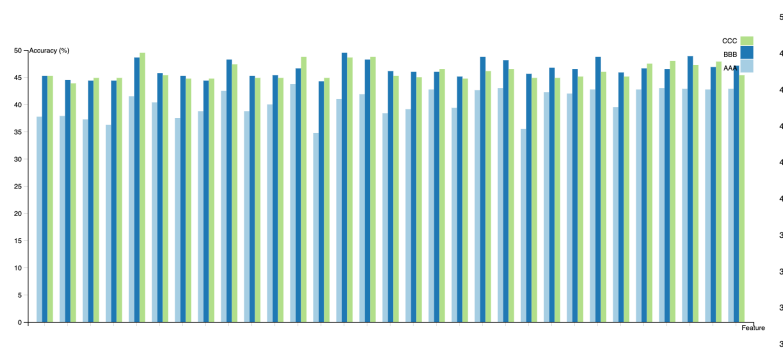

(a) Bar chart.

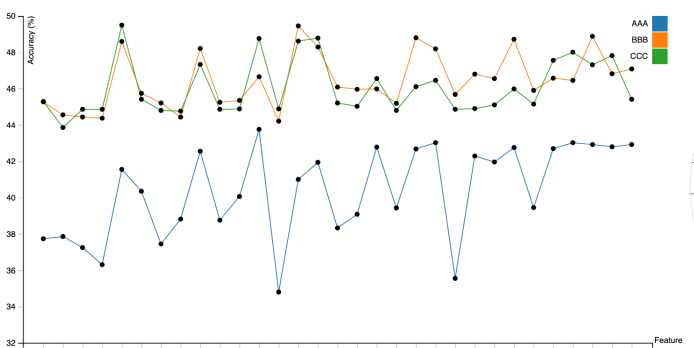

(b) Line chart.

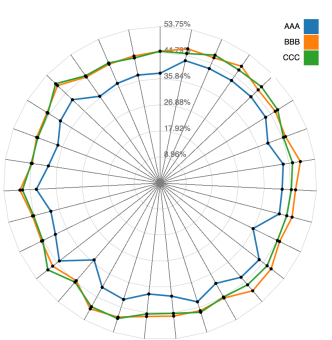

(c) Radar chart.

Figure 8. Comparison of three ML algorithms for the same data set with three visualisation approaches. 


\subsection{Procedure and Data Collection}

The study was conducted in a lab environment using a Macbook Pro with 13-inch display of resolution $2560 \times 1600$. The procedure of the study is described as follows: Tutorial slides on the study were firstly presented to participants in order to inform them of the concepts and operations during the study. A training task was then conducted to practice interactions. After that, the formal tasks were conducted with different visualisations. During the study, different visualisations as described in the previous section were displayed to participants one-by-one in random order. For each visualisation, participants were firstly required to find the ML model that provides the best or worst performance by selecting the visual elements in the visualisation (we call this the selection task). This is more akin to what analysts carry out with real data sets. After the selection task, the participants were asked to answer different questions as described below on the task and visualisation. At the end of the study, participants were asked to provide their feedback with respect to using the charts and some personal details such as gender, age, and working topics.

After the selection task of each visualisation, the participants were asked to answer questions related to comparison, feature importance, feature identification, visual complexity, and mental effort on the visualisation using 9-point Likert scales (comparison, feature importance, and feature identification: $1=$ least easiness, $9=$ most easiness; visual complexity: 1 = least complex, $9=$ most complex; mental effort: $1=$ least effort, $9=$ most effort). At the end of all visualisation tasks, the participants were also asked to answer in a questionnaire which visualisation helped users to more easily compare ML performance of different features and which visualisation helps users to more easily compare the ML performance of different ML algorithms, respectively.

\subsection{Results}

In this section, for the evaluation of each metric, we firstly performed one-way ANOVA test and then followed it up with post-hoc analysis using $t$-tests (with a Bonferroni correction under a significance level set at $p<\frac{0.05}{4}=0.013$, based on the fact that we had four visualisation types to test) in order to analyze the differences in participant responses of each metric. Each metric value was normalised with respect to each subject in order to minimise individual differences in rating behavior (refer to Equation (2)):

$$
T_{i}^{N}=\frac{T_{i}-T_{i}^{\min }}{T_{i}^{\max }-T_{i}^{\min }}
$$

where $T_{i}$ and $T_{i}^{N}$ are the original metric rating and the normalised metric rating, respectively, from the participant $i$, and $T_{i}^{\min }$ and $T_{i}^{\max }$ are the minimum and maximum of metric ratings, respectively, from the participant $i$ in all of his/her tasks. The time spent on the selection tasks is also normalised in a similar manner as the other five metrics.

Figure 9 shows mean normalised metric values for different visualisation types.

Comparison easiness. The one-way ANOVA test provided significant differences in comparison easiness among four visualisation types $(F(3,84)=3.067, p<0.03)$ (see Figure $9 a)$. However, the post-hoc $t$-tests only found that the line chart was significantly easier for comparing the performance of different ML models than compared to the radar chart $(t=2.813, p<0.007)$. The result shows that RadialNet did not help users in increasing easiness in comparing the performance of different ML models, which is not as we expected, but a trend shows the higher ratings in comparison easiness for RadialNet than the bar chart and radar chart (see Figure 9a). This may be because of the relatively small number of participants used for the study.

Feature identification. One-way ANOVA test found significant differences in easiness of feature identification among four visualisation types $(F(3,84)=6.108, p<0.001)$ (see Figure $9 b$ ). The post-hoc $t$-tests found that RadialNet was significantly easier for identifying features related to models than all of the other three visualisation 
types (line chart: $t=3.296, p<0.002$; bar chart: $t=3.393, p<0.002$; radar chart: $t=4.089, p=0.000)$. This is because that users can obtain features and performance related to an ML model directly from connected visual elements in RadialNet, while users need to move the mouse to visual elements of each model to inspect related features and performance in the other three visualisations.

Feature importance. There were significant differences found in the ease of identifying feature importance among four visualisation types by one-way ANOVA test $(F(3,84)=14.481, p=0.000)$ (see Figure 9c). The post-hoc $t$-tests found that RadialNet was significantly easier for identifying feature importance than all of the other three visualisation types (line chart: $t=4.878, p=0.000$; bar chart: $t=5.320, p=0.000$; radar chart: $t=7.678, p=0.000)$. The results suggest the obvious advantage of RadialNet over the other three visualisation types for feature importance identifications.

Visual complexity. One-way ANOVA test found significant differences in visual complexity among the four visualisation types $(F(3,84)=20.254, p=0.000)$ (see Figure $9 \mathrm{~d}$ ). The post-hoc $t$-tests found that RadialNet was significantly more complex than all of the other three visualisation types (line chart: $t=7.032, p=0.000$; bar chart: $t=6.001, p=0.000$; radar chart: $t=3.710, p<0.001)$. It was also observed that radar chart was significantly more complex than the line chart $(t=3.383, p<0.002)$.

Mental effort. There were significant differences found in mental effort among the four visualisation types by one-way ANOVA test $(F(3,84)=8.757, p=0.000$ ) (see Figure 9e). The post-hoc tests found that line chart took significantly less effort than the other three visualisation types (bar chart: $t=3.722, p<0.001$; radar chart: $t=4.981, p=0.000$; RadialNet: $t=5.562, p=0.000)$. RadialNet did not show significant differences in mental effort with radar chart and bar chart.

Time spent. One-way ANOVA test found significant differences in time spent in the selection of the best/worst model task among the four visualisation types $(F(3,84)=5.301$, $p<0.002$ ) (see Figure 9f). The post-hoc tests found that users spent significantly more time in RadialNet than in both line chart $(t=3.286, p<0.002)$ and bar chart $(t=3.111, p<0.003)$, respectively.

When four types of visualisation were used to compare performance of different ML algorithms for a given data set, it was found that line chart was easier to compare performance of different ML algorithms followed by RadialNet despite no significant differences found in easiness. This could be because of the relatively small number of participants in this study. However, RadialNet can reveal the importance of features while others do not when comparing the performance of different ML algorithms.

We also collected participants' feedback after completing all tasks by each participant. Overall, all participants believed that "RadialNet is the most effective visualisation in identifying feature importance compared with other three approaches." Some participants suggested to "enlarge the size of RadialNet with the increase of number of features." Participants agreed that "RadialNet is more efficient to help users focus their attention to find visual elements of interest." Figures 10 and 11 show heat maps on four visualisations recorded by an SMI eye-tracker from a participant during the selection task period, respectively. Heat maps reveal the focus of attention by colours indicating the amount of time eyes stay focused on a particular area in the visualisation; the redder it is, the longer period of time the eyes stayed focused. Figures 10 and 11 suggest that the user's attention in RadialNet was more focused on two model lines with high performance (wide red lines), while it was much more scattered among different points in the three other visualisations. 


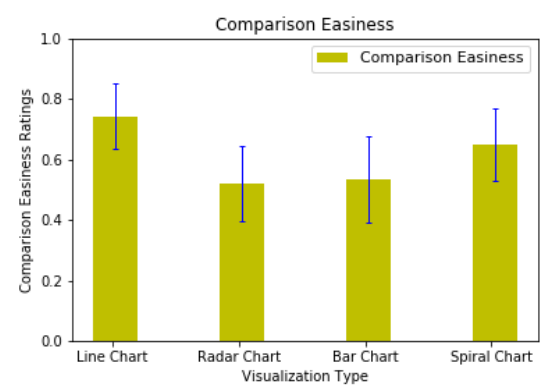

(a) Comparison easiness.

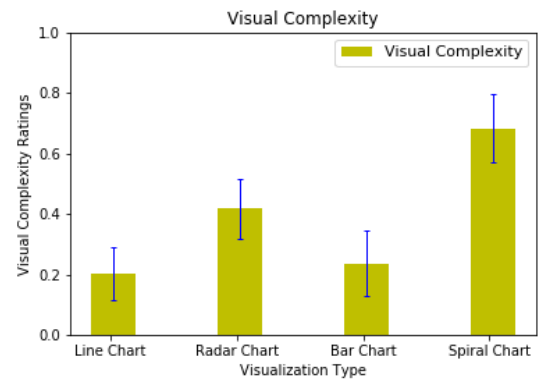

(d) Visual complexity.

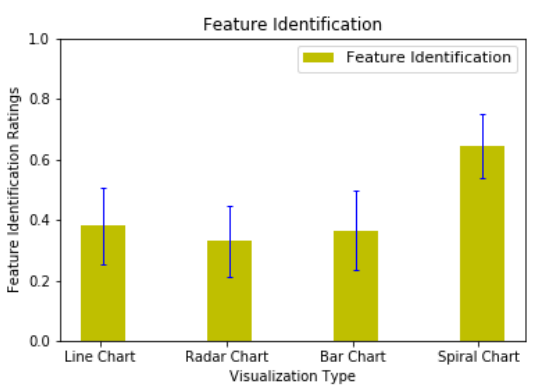

(b) Feature identification.

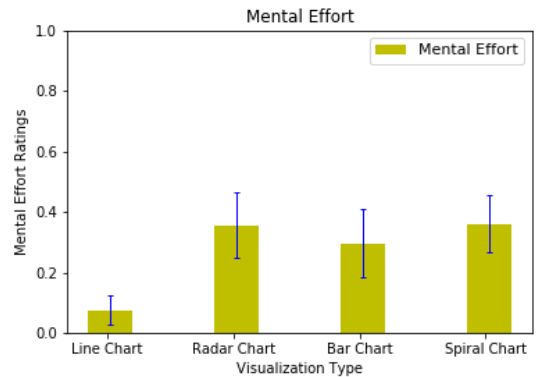

(e) Mental effort.

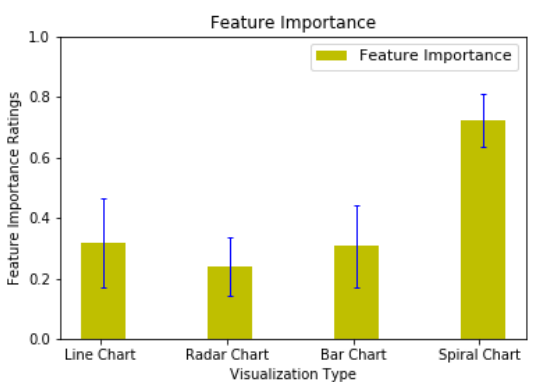

(c) Feature importance.

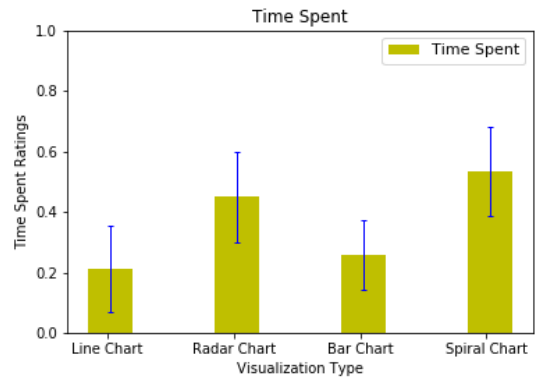

(f) Time spent.

Figure 9. Comparison of mean normalized metrics for different visualisation types.

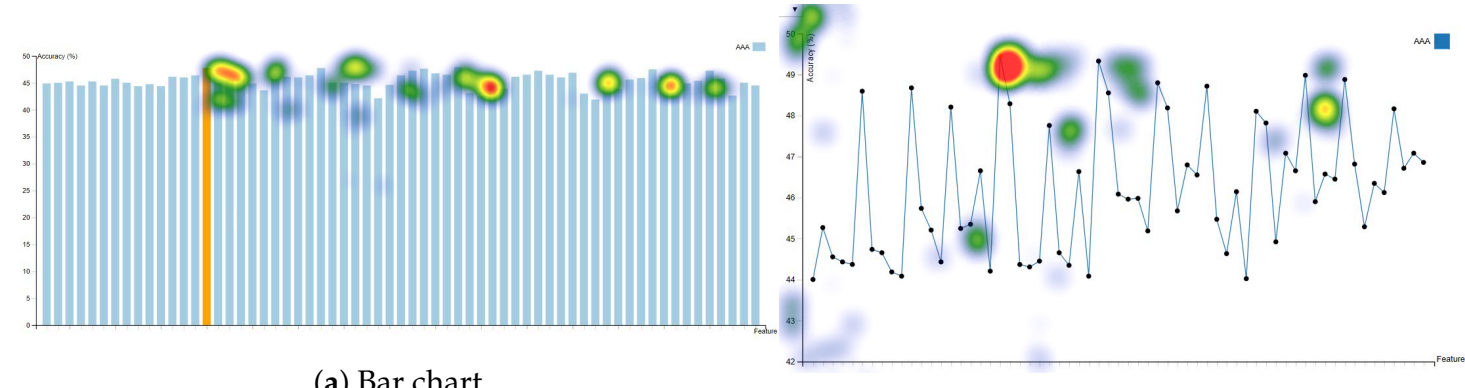

(b) Line chart

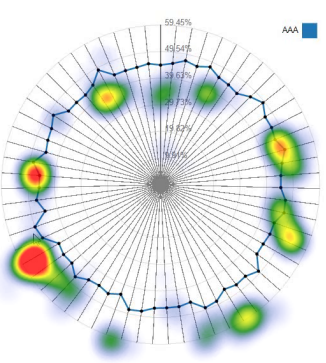

(c) Radar chart

Figure 10. Heat maps of bar chart, line chart, and radar chart.
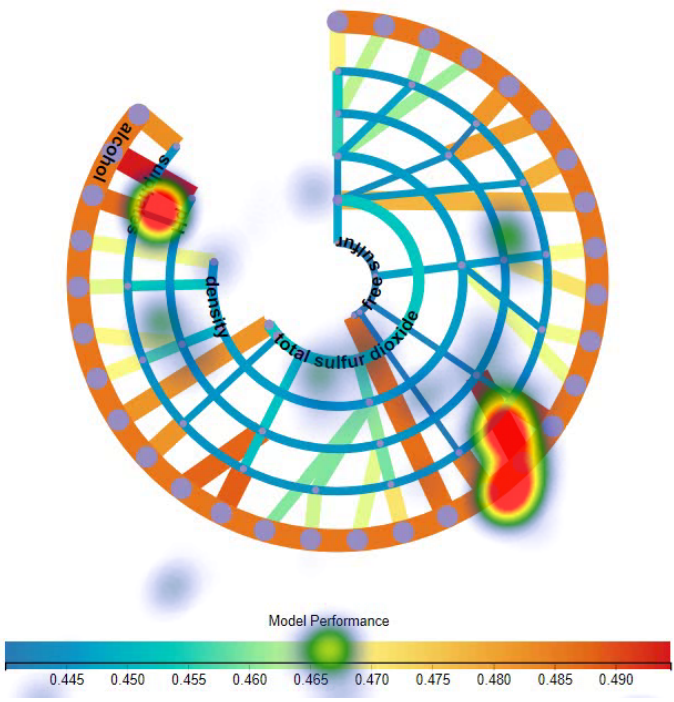

Figure 11. Heat map of RadialNet. 
Overall, we can say that RadialNet shows significant advantages in identifying features and performance related to specific models, and it easily reveals the importance of features compared with the other three visualisation types. Despite these advantages, the mental effort and time spent in RadialNet did not show much differences from other visualisations such as radar chart.

\section{Discussion}

This study proposed a novel visualisation approach for comparing variables with a different number of dependents. Data information is encoded with colour, line width, and structure of visualisation for revealing insights from data. The experimental results showed that RadialNet has advantages in identifying features related to specific models as well as directly revealing the importance of features for ML explanations. Distinct from conventional feature importance evaluations based on complex computing algorithms (such as by simulating lack of knowledge about the values of the feature(s) [40] or by mean decrease impurity, which is defined as the total decrease in node impurity averaged over all trees of the ensemble in Random Forest [41]), RadialNet allows users to estimate feature importance directly from visualisation by checking lines connected to the feature arc. The consistent large line width of these lines with colours on the right-hand side of the colour scale indicate the high importance of the feature to modelling.

RadialNet is more compact and can show more information in a limited amount of space compared with the three other visualisation types. Moreover, the compactness of RadialNet can also be controlled by changing its spanning angle dynamically (see the attached video with this paper). However, RadialNet will be much complex when the number of features is high. This could be compensated with large scale visualisation facilities. For example, we have a 360-degree interactive data visualisation facility set to change the way we view and interact with data. Viewers stand in the middle of a large cylindrical screen that is four metres high and ten metres in diameter. A high performance computer graphics system drives six 3D-stereo video projectors that are edge-blended to create a seamless three-dimensional panorama. Picture clarity is made possible from an image with $20,000 \times 1200$ pixels. This facility can be used to present RadialNet with a large number of ML models for effective interactions. Figure 12 shows an example of RadialNet displayed with around 60-degree field of view in the facility.

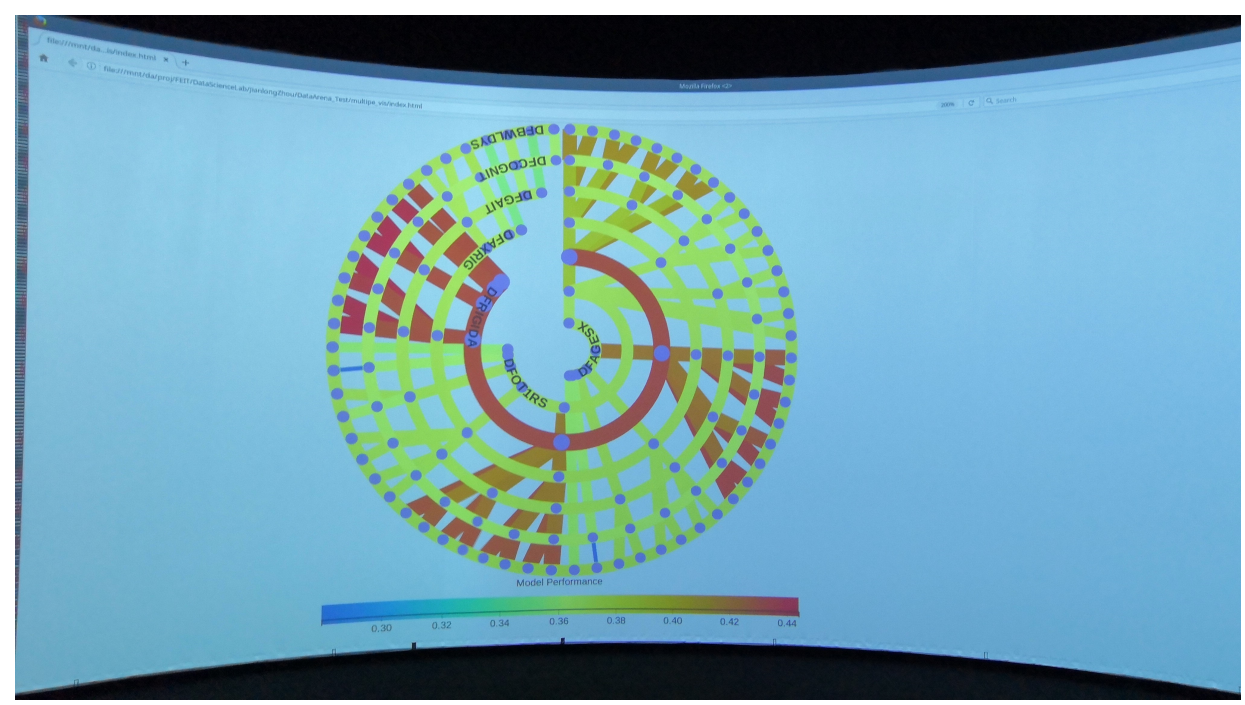

Figure 12. RadialNet displayed in our large scale visualisation facility.

This paper used the exploration of the performance of ML models based on different feature groups from a given data set as a case study to demonstrate the powerfulness of RadialNet in visualising data with complex relations. The RadialNet can also be generalised to other applications where similar relations need to be explored. 


\section{Conclusions}

This paper presented RadialNet Chart, a novel visualisation approach for comparing ML models with a different number of features while revealing implicit dependent relations. RadialNet is developed to address the challenges faced in comparing a large amount of ML models with each dependent on a dynamic number of features. It is implemented by representing ML models and features with lines and arcs, respectively, which in turn are generated by a recursive function and a feature path concept. We presented our design criteria and described the algorithms for generating the chart. Two case studies were also presented with representative data sets, and an experiment was conducted for evaluating the effectiveness of the RadialNet. Our case studies showed that the proposed visualisation can help users to easily locate target models and important features. Furthermore, the user study revealed that in comparison with other commonly used visualisation approaches, RadialNet is more efficient for helping users to focus their attention in finding visual elements of interest. It is also more compact for showing more information in a limited amount of space. Our research provides an effective visualisation approach for representing data with complex relations. It is specifically helpful for users to find optimal machine learning models and discern feature importance visually and directly but not through complex algorithmic calculations for ML explanations.

Author Contributions: Conceptualization, J.Z. and F.C.; methodology, J.Z. and W.H.; software, J.Z. and W.H.; formal analysis, J.Z. and F.C.; investigation, J.Z.; resources, W.H.; data curation, J.Z.; writing—original draft preparation, J.Z. and W.H.; writing-review and editing, F.C.; visualization, J.Z. and W.H.; supervision, F.C.; project administration, F.C. All authors have read and agreed to the published version of the manuscript.

Funding: This research received no external funding.

Institutional Review Board Statement: The study was approved by the Human Research Ethics Committee (HREC) of University of Technology Sydney (ETH19-3400, January 2019).

Informed Consent Statement: Informed consent was obtained from all subjects involved in the study. Data Availability Statement: Not applicable.

Conflicts of Interest: The authors declare no conflicts of interest.

\section{References}

1. Card, S.K.; Mackinlay, J.D.; Shneiderman, B. Readings in Information Visualization: Using Vision to Think; Morgan Kaufmann: San Francisco, CA, USA; 1999.

2. Becker, B.; Kohavi, R.; Sommerfield, D. Visualizing the Simple Bayesian Classifier. In Information Visualization in Data Mining and Knowledge Discovery; Fayyad, U., Grinstein, G.G., Wierse, A., Eds.; Morgan Kaufmann: San Francisco, CA, USA 2001; pp. 237-249.

3. Talbot, J.; Lee, B.; Kapoor, A.; Tan, D.S. EnsembleMatrix: Interactive visualization to support machine learning with multiple classifiers. In Proceedings of the SIGCHI Conference on Human Factors in Computing Systems, Boston, MA, USA, 4-9 April 2009; pp. 1283-1292.

4. Wu, A.; Wang, Y.; Shu, X.; Moritz, D.; Cui, W.; Zhang, H.; Zhang, D.; Qu, H. Survey on Artificial Intelligence Approaches for Visualization Data. arXiv 2021, arXiv:2102.01330.

5. Viegas, F.; Wattenberg, M. Visualization: The secret weapon for machine learning. In Keynote in EuroVis 2017; Barcelona, Spain; 2017. Available online: https:/ / youtu.be/E70lG9-HGEM (accessed on 15 August 2021)

6. Aigner, W.; Miksch, S.; Schumann, H.; Tominski, C. Visualization of Time-Oriented Data; Human-Computer Interaction Series; Springer: Berlin/Heidelberg, Germany, 2011.

7. Gleicher, M. Considerations for Visualizing Comparison. IEEE Trans. Vis. Comput. Graph. 2018, 24, 413-423. [CrossRef]

8. Law, P.; Basole, R.C.; Wu, Y. Duet: Helping Data Analysis Novices Conduct Pairwise Comparisons by Minimal Specification. IEEE Trans. Vis. Comput. Graph. 2019, 25, 427-437. [CrossRef] [PubMed]

9. Ondov, B.D.; Jardine, N.; Elmqvist, N.; Franconeri, S. Face to Face: Evaluating Visual Comparison. IEEE Trans. Vis. Comput. Graph. 2019, 25, 861-871. [CrossRef]

10. Zhou, J.; Huang, W.; Chen, F. A Radial Visualisation for Model Comparison and Feature Identification. In Proceedings of the IEEE PacificVis 2020, Tianjin, China, 14-17 April 2020; pp. 226-230.

11. Tian, Z.; Zhai, X.; van Driel, D.; van Steenpaal, G.; Espadoto, M.; Telea, A. Using multiple attribute-based explanations of multidimensional projections to explore high-dimensional data. Comput. Graph. 2021, 98, 93-104. [CrossRef] 
12. Guo, H.; Xiao, H.; Yuan, X. Scalable Multivariate Volume Visualization and Analysis Based on Dimension Projection and Parallel Coordinates. IEEE Trans. Vis. Comput. Graph. 2012, 18, 1397-1410.

13. Kim, S.; Dong, Z.; Xian, H.; Upatising, B.; Yi, J.S. Does an Eye Tracker Tell the Truth about Visualizations? Findings while Investigating Visualizations for Decision Making. IEEE Trans. Vis. Comput. Graph. 2012, 18, 2421-2430. [CrossRef]

14. Zhou, J.; Sun, J.; Chen, F.; Wang, Y.; Taib, R.; Khawaji, A.; Li, Z. Measurable Decision Making with GSR and Pupillary Analysis for Intelligent User Interface. ACM Trans. Comput.-Hum. Interact. 2015, 21, 33. [CrossRef]

15. Roberts, J.C. State of the Art: Coordinated \& Multiple Views in Exploratory Visualization. In Proceedings of the Fifth International Conference on Coordinated and Multiple Views in Exploratory Visualization (CMV '07), Zurich, Switzerland, 2 July 2007; pp. 61-71.

16. Langner, R.; Horak, T.; Dachselt, R. VisTiles: Coordinating and Combining Co-located Mobile Devices for Visual Data Exploration. IEEE Trans. Vis. Comput. Graph. 2018, 24, 626-636. [CrossRef]

17. Koytek, P.; Perin, C.; Vermeulen, J.; André, E.; Carpendale, S. MyBrush: Brushing and Linking with Personal Agency. IEEE Trans. Vis. Comput. Graph. 2018, 24, 605-615. [CrossRef]

18. Sarikaya, A.; Gleicher, M. Scatterplots: Tasks, Data, and Designs. IEEE Trans. Vis. Comput. Graph. 2018, 24, 402-412. [CrossRef]

19. Yuan, J.; Chen, C.; Yang, W.; Liu, M.; Xia, J.; Liu, S. A survey of visual analytics techniques for machine learning. Comput. Vis. Media 2021, 7, 3-36. [CrossRef]

20. Chatzimparmpas, A.; Martins, R.M.; Jusufi, I.; Kucher, K.; Rossi, F.; Kerren, A. The State of the Art in Enhancing Trust in Machine Learning Models with the Use of Visualizations. Comput. Graph. Forum 2020, 39, 713-756. [CrossRef]

21. Cashman, D.; Humayoun, S.R.; Heimerl, F.; Park, K.; Das, S.; Thompson, J.; Saket, B.; Mosca, A.; Stasko, J.T.; Endert, A.; Gleicher, M.; Chang, R. A User-based Visual Analytics Workflow for Exploratory Model Analysis. Comput. Graph. Forum 2019, 38, 185-199. [CrossRef]

22. Ankerst, M.; Elsen, C.; Ester, M.; Kriegel, H.P. Visual classification: An interactive approach to decision tree construction. In Proceedings of the fifth ACM SIGKDD International Conference on Knowledge Discovery and Data Mining, San Diego, CA, USA, 15-18 August 1999; pp. 392-396.

23. Caragea, D.; Cook, D.; Honavar, V.G. Gaining insights into support vector machine pattern classifiers using projection-based tour methods. In Proceedings of the Seventh ACM SIGKDD International Conference on Knowledge Discovery and Data Mining, San Diego, CA, USA, 26-29 August 2001; pp. 251-256.

24. Erra, U.; Frola, B.; Scarano, V. An Interactive Bio-inspired Approach to Clustering and Visualizing Datasets. In Proceedings of the 15th International Conference on Information Visualisation 2011, London, UK, 13-15 July 2011; pp. 440-447.

25. Paiva, J.G.; Florian, L.; Pedrini, H.; Telles, G.; Minghim, R. Improved Similarity Trees and their Application to Visual Data Classification. IEEE Trans. Vis. Comput. Graph. 2011, 17, 2459-2468. [CrossRef] [PubMed]

26. Guo, Z.; Ward, M.O.; Rundensteiner, E.A. Nugget Browser: Visual Subgroup Mining and Statistical Significance Discovery in Multivariate Datasets. Proceedings of the 15th International Conference on Information Visualisation, London, UK, 13-15 July 2011; pp. 267-275.

27. Zhou, J.; Khawaja, M.A.; Li, Z.; Sun, J.; Wang, Y.; Chen, F. Making Machine Learning Useable by Revealing Internal States Update-A Transparent Approach. Int. J. Comput. Sci. Eng. 2016, 13, 378-389. [CrossRef]

28. Amershi, S.; Chickering, M.; Drucker, S.M.; Lee, B.; Simard, P.; Suh, J. ModelTracker: Redesigning Performance Analysis Tools for Machine Learning. In Proceedings of the 33rd Annual ACM Conference on Human Factors in Computing Systems, Seoul, Korea, 18-23 April 2015; pp. 337-346.

29. Chen, D.; Bellamy, R.K.E.; Malkin, P.K.; Erickson, T. Diagnostic visualization for non-expert machine learning practitioners: A design study. In Proceedings of the 2016 IEEE Symposium on Visual Languages and Human-Centric Computing (VL/HCC), Cambridge, UK, 4-8 September 2016; pp. 87-95.

30. Wongsuphasawat, K.; Smilkov, D.; Wexler, J.; Wilson, J.; Mané, D.; Fritz, D.; Krishnan, D.; Viégas, F.B.; Wattenberg, M. Visualizing Dataflow Graphs of Deep Learning Models in TensorFlow. IEEE Trans. Vis. Comput. Graph. 2018, 24, 1-12. [CrossRef] [PubMed]

31. Qi, J.; Bloemen, V.; Wang, S.; van Wijk, J.; van de Wetering, H. STBins: Visual Tracking and Comparison of Multiple Data Sequences Using Temporal Binning. IEEE Trans. Vis. Comput. Graph. 2020, 26, 1054-1063. [CrossRef]

32. Pflüger, H.; Thom, D.; Schütz, A.; Bohde, D.; Ertl, T. VeCHArt: Visually Enhanced Comparison of Historic Art Using an Automated Line-Based Synchronization Technique. IEEE Trans. Vis. Comput. Graph. 2020, 26, 3063-3076. [CrossRef]

33. Cutura, R.; Aupetit, M.; Fekete, J.D.; Sedlmair, M. Comparing and Exploring High-Dimensional Data with Dimensionality Reduction Algorithms and Matrix Visualizations. In Proceedings of the International Conference on Advanced Visual Interfaces, Ischia Island, Italy, 28 September-2 October 2020.

34. Heimerl, F.; Kralj, C.; Moller, T.; Gleicher, M. embComp: Visual Interactive Comparison of Vector Embeddings. IEEE Trans. Vis. Comput. Graph. 2020. [CrossRef] [PubMed]

35. Biran, O.; Cotton, C. Explanation and Justification in Machine Learning: A Survey. In Proceedings of the 2017 IJCAI Explainable AI Workshop, Melbourne, Australia, 19-25 August 2017; pp. 8-13.

36. Bostock, M.; Ogievetsky, V.; Heer, J. D3 Data-Driven Documents. IEEE Trans. Vis. Comput. Graph. 2011, 17, 2301-2309. [CrossRef] [PubMed]

37. Dua, D.; Karra Taniskidou, E. UCI Machine Learning Repository. 2017. Available online: https://archive.ics.uci.edu/ml/index.php (accessed on 1 February 2020). 
38. Prakash, N.; Caspell-Garcia, C.; Coffey, C.; Siderowf, A.; Tanner, C.M.; Kieburtz, K.; Mollenhauer, B.; Galasko, D.; Merchant, K.; Foroud, T.; et al.. Feasibility and safety of lumbar puncture in the Parkinson's disease research participants: Parkinson's Progression Marker Initiative (PPMI). Parkinsonism Relat. Disord. 2019, 62, 201-209 [CrossRef] [PubMed]

39. Huang, W. Establishing aesthetics based on human graph reading behavior: Two eye tracking studies. Pers. Ubiquitous Comput. 2013, 17, 93-105. [CrossRef]

40. Robnik-Sikonja, M.; Kononenko, I.; Strumbelj, E. Quality of Classification Explanations with PRBF. Neurocomputing 2012, 96, 37-46. [CrossRef]

41. Breiman, L.; Friedman, J.; Olshen, R.; Stone, C. Classification and Regression Trees; Wadsworth and Brooks: Monterey, CA, USA, 1984 